OPEN ACCESS

Edited by:

Inge Bauer,

University Hospital of

Düsseldorf, Germany

Reviewed by:

Enrico Calzia,

University of UIm, Germany

Michael A. Dubick,

United States Army Institute of

Surgical Research, United States

*Correspondence:

Raghavan Pillai Raju

rraju@augusta.edu

Specialty section:

This article was submitted to

Intensive Care Medicine and Anesthesiology,

a section of the journal

Frontiers in Medicine

Received: 28 August 2019 Accepted: 18 November 2019 Published: 17 December 2019

Citation:

Chu X, Schwartz R, Diamond MP and Raju RP (2019) A Combination

Treatment Strategy for Hemorrhagic Shock in a Rat Model Modulates Autophagy. Front. Med. 6:281. doi: 10.3389/fmed.2019.00281

\section{A Combination Treatment Strategy for Hemorrhagic Shock in a Rat Model Modulates Autophagy}

\author{
Xiaogang Chu ${ }^{1}$, Richard Schwartz ${ }^{2}$, Michael P. Diamond ${ }^{3}$ and Raghavan Pillai Raju ${ }^{1 *}$ \\ ${ }^{1}$ Department of Pharmacology and Toxicology, Medical College of Georgia, Augusta University, Augusta, GA, United States, \\ ${ }^{2}$ Emergency Medicine, Medical College of Georgia, Augusta University, Augusta, GA, United States, ${ }^{3}$ Department of \\ Obstetrics and Gynaecology, Medical College of Georgia, Augusta University, Augusta, GA, United States
}

Hemorrhagic shock leads to whole body hypoxia and nutrient deprivation resulting in organ dysfunction and mortality. Previous studies demonstrated that resveratrol, dichloroacetate, and niacin improve organ function and survival in rats following hemorrhagic shock injury $(\mathrm{HI})$. We hypothesized that a combinatorial formula that collectively promotes survival will decrease the dose of individual compounds toward effective therapy for HI. Sprague-Dawley rats were subjected to $\mathrm{HI}$ by withdrawing $60 \%$ blood volume. NiDaR (Niacin-Dichloroacetate-Resveratrol; 2 mg/kg dose of each) or vehicle was administered following the shock in the absence of fluid resuscitation, and survival monitored. In order to study alterations in molecular mediators, separate groups of rats were administered $\mathrm{NiDaR}$ or vehicle along with resuscitation fluid, following $\mathrm{HI}$. We observed significant improvement $(p<0.05)$ in survival following $\mathrm{HI}$ in animals that received NiDaR, in the absence of fluid resuscitation. In NiDaR treated animals that received resuscitation fluid, MAP was significantly increased compared to Veh-treated rats. HI-induced increase in systemic IL-6 levels and tissue expression of $I L-6, I L-10, I L-1 \beta$, and $I L-18$ genes in the heart were attenuated with $\mathrm{NiDaR}$ treatment. NiDaR promoted autophagy following $\mathrm{HI}$ as demonstrated by reduced p-mTOR, increased p-ULK1 and p-Beclin. The combinatorial formula, NiDaR, reduced inflammation, promoted autophagy, and reduced doses of individual compounds used, and may be more effective in genetically heterogeneous population. In conclusion our experiments demonstrated that the combinatorial drug treatment has salutary effect in rats following $\mathrm{HI}$.

Keywords: trauma, hemorrhage, shock, autophagy, mitochondria

\section{INTRODUCTION}

Trauma is the leading cause of death in the $1-45$ years age group $(1,2)$. Hemorrhage is a common cause of death following traumatic injuries (1-3). There is a lack of consensus on the use of any specific resuscitation strategy or adjuncts to fluid resuscitation following severe hemorrhage and therefore there is a need to develop suitable methods in the treatment of hemorrhagic shock (4). The availability of adjuncts that complement limited or no resuscitation may play critical role in prolonging life following hemorrhagic shock.

Our experiments using a rat model of hemorrhagic shock injury (HI) demonstrated that in the absence of volume replacement, a protracted metabolic homeostasis may be achieved using 
agents that potentiate cellular energetics (5-7). Cellular energetics is an important determining factor in maintaining homeostatic balance (7-9). Our studies unequivocally showed that the naturally occurring SIRT1 activator, resveratrol (RSV), can prolong survival in experimental rats following $\mathrm{HI}$ in the absence of fluid resuscitation (5). SIRT1 deacetylates target proteins in a $\mathrm{NAD}^{+}$dependent mechanism. The effect of resveratrol in improving organ function in experimental animals subjected to hemorrhagic shock has been described by many laboratories (10-14).

We also observed prolonged survival following HI when the rats were treated with niacin (15). Niacin metabolizes to produce $\mathrm{NAD}^{+}$, increases SIRT1 activity and enhance mitochondrial function (16). Our continuing experiments seeking a role for cellular energetics in modulating outcome following HI demonstrated the significance of activation of pyruvate dehydrogenase (Pdh), a key protein connecting glycolysis and citric acid cycle in HI. We found that dichloroacetate (DCA), a classic inhibitor of pyruvate dehydrogenase kinase (Pdk), significantly improved organ function and survival following HI (6). Pdk is activated in tissue hypoxia resulting in inhibition of Pdh thereby reducing the availability of acetyl CoA to citric acid cycle. Therefore, inhibition of Pdk by DCA activates Pdh and citric acid cycle with resultant increase in ATP production.

The three compounds, resveratrol, DCA and niacin, agents that are known to enhance autophagy and mitochondria function $(5,6,15,17-22)$, were found to have optimal effect when used at a dose of $10-25 \mathrm{mg} / \mathrm{Kg}$ body weight in the rats. We hypothesized that a combinatorial formula that collectively promotes survival will decrease the dose of individual compounds toward improving survival after HI. In this study we tested whether a combination of resveratrol, DCA and niacin (NiDaR) can be used in reduced individual doses with similar outcome, and examined the regulation of autophagy following treatment with $\mathrm{NiDaR}$.

\section{MATERIALS AND METHODS}

\section{Hemorrhage Injury Procedure}

The animals were subjected to sham or $\mathrm{HI}$ procedure as described before $(5,6)$. Briefly, the animals were anesthetized with isoflurane (Henry Schein, Dublin, $\mathrm{OH}$, USA) and a midline laparotomy $(3 \mathrm{~cm})$ was performed to induce soft tissue trauma. Femoral arteries and one femoral vein were cannulated (PE50 tubing), for bleeding, blood pressure monitoring, and fluid resuscitation. Surgical sites were bathed with bupivacaine. Sham animals did not undergo hemorrhage or fluid resuscitation. Hemorrhagic shock injury was induced by bleeding rapidly to a MAP of $40 \pm 5 \mathrm{mmHg}$ in the first $10 \mathrm{~min}$ and bleeding continued for $45 \mathrm{~min}$ to remove $60 \%$ of the total blood volume. The animals were maintained in the state of shock by maintaining the low MAP for another $45 \mathrm{~min}$. Two models were used. Model 1: In this model animals were resuscitated with fluid. The fluid resuscitation was carried out with Ringer lactate (RL; twice the volume of shed blood) following hemorrhage and shock, and lasted for $1 \mathrm{~h}$. Vehicle (DMSO; $120 \mu \mathrm{L} /$ rat) or NiDaR was administered at $10 \mathrm{~min}$ from the start of resuscitation. The animals were euthanized at $2 \mathrm{~h}$ after the end of fluid resuscitation and tissues saved. Model 2: Animals in this group were not resuscitated with fluid. This model was used for short-term survival studies to determine the effect of NiDaR on length of survival in the absence of fluid resuscitation. NiDaR or DMSO was administered (100 $\mu \mathrm{L}$ with 300-400 $\mu \mathrm{l} \mathrm{RL}$ to flush the catheter) intravenously at the end of the shock period and survival observed. Euthanasia was performed by overdose of isoflurane followed by removal of heart.

\section{Immunoblotting Analysis}

Immunoblotting procedures were performed as we described previously. Briefly, the heart samples were homogenized in the lysis buffer. Lysates were clarified at 12,000 g for $10 \mathrm{~min}$ at $4^{\circ} \mathrm{C}$, and protein concentrations were determined by the Bradford protein assay (Bio-Rad Laboratories, Hercules, CA). Equal amounts of protein were loaded onto $8-12 \%$ SDSPAGE, transferred onto polyvinylidene difluoride membranes, probed with the indicated primary antibody and the appropriate secondary antibody conjugated with horseradish peroxidase (Cell Signaling, Danvers, MA), and the immune complexes were detected by standard methods. The antibodies used in this study were as follows: GAPDH (Cell Signaling, Danvers, MA), p-P65 (Cell Signaling, Danvers, MA), P65 (Cell Signaling, Danvers, MA), p-ULK1 (Cell Signaling, Danvers, MA), ULK1 (Cell Signaling, Danvers, MA), p-Beclin1 (Cell Signaling, Danvers, MA), Beclin1 (Cell Signaling, Danvers, MA), LC3 (Cell Signaling, Danvers, MA), p-MTOR (Cell Signaling, Danvers, MA), MTOR (Cell Signaling, Danvers, MA), NLRP3 (Abcam, Cambridge, MA), SQSTM1 (Abcam, Cambridge, MA). HRP linked antirabbit or anti-mouse IgG second antibodies (Cell Signaling, Danvers, MA).

\section{Real-Time Polymerase Chain Reaction}

The heart cytokines were tested by real time PCR. Total RNA was isolated using TRIZOL reagent (Thermo Fisher, Waltham, MA) and cDNA was synthesized using ImPromII $^{\mathrm{TM}}$ Reverse Transcription System (Promega, Madison, WI). Realtime PCR was performed as described previously. The sequences of the primers used were: IL-1 $\beta$ : Forward: CCC TGCAGCTGGAGAGTGTGG, Reverse: TGTGCTCTGCTT GAGAGGTGCT, IL-18: Forward: CAGACCACTTTGGCA GACTTCACT, Reverse: GGATTCGTTGGCTGTTCGGTC G, IL-6: Forward: GAGCCCACCAGGAACGAAA, Reverse: AACTGGCTGGAAGTCTCTTGC; IL-10: Forward: TGCGAC GCTGTCATCGATTT, Reverse: GTAGATGCCGGGTGGTTC AA; $\beta$-actin: Forward: AGTACCCCATTGAACACG; Reverse: AATGCCAGTGGTACGACC. The results are expressed after normalizing to the values obtained for samples in control group.

\section{Lactate Assay}

Heparinized blood samples were obtained prior to euthanasia. The plasma was separated and lactate levels were measured using a Lactate Assay Kit (Sigma, St. Louis, MO) according to the manufacturer's protocol. 


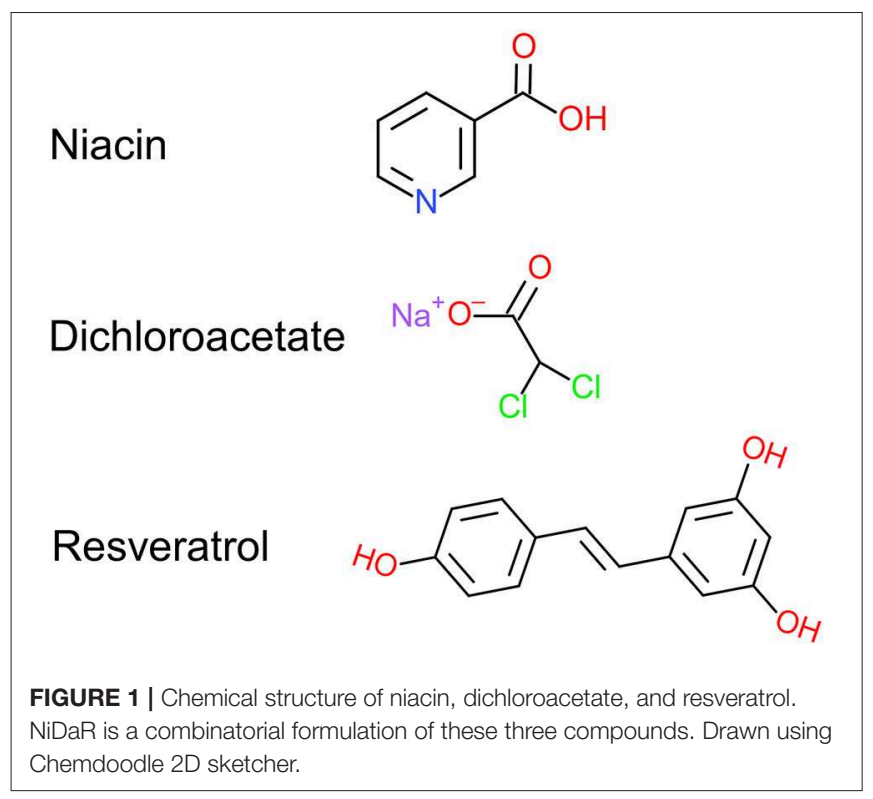

\section{IL-6 Assay}

Plasma IL-6 concentrations were determined using an IL-6 Rat ELISA Kit (Thermo Fisher, Waltham, MA) according to the manufacturer's protocol.

\section{Statistics}

Data are presented as mean \pm S.E.M. Student $t$-test or Oneway ANOVA, was used for statistical analysis using Prism 6 (GraphPad Software). $p<0.05$ was considered to be statistically significant. The primary outcome in the non-resuscitation study was survival duration. When MAP reduced to below $30 \mathrm{~mm}$ $\mathrm{Hg}$ the animals were euthanized, as death could not be an endpoint (6).

\section{RESULTS}

\section{Survival Study Using Combination Dose}

In this study we tested whether reduced doses of Niacin, Dichloroacetate, and Resveratrol (NiDaR) administered as a combination can improve the outcome following HI (Figure 1). Each of the constituents in NiDaR was used at $2 \mathrm{mg} / \mathrm{Kg}$ dose. The mixture was administered intravenously either at $10 \mathrm{~min}$ after the start of fluid resuscitation or at the end of shock period in the subset of animals that did not receive fluid resuscitation. As shown in Figure 2, the combination dose significantly improved survival in the absence of fluid resuscitation. The animals were euthanized when the MAP reached below $30 \mathrm{mmHg}$ as death cannot be an endpoint. It may be noted that when fluid resuscitation is not performed following hemorrhagic shock, in our model, MAP reaching $30 \mathrm{mmHg}$ has been observed to be a point of no return for the rats.

\section{The Effect of NiDaR Treatment on MAP and Blood Lactate}

As shown in Figure 3A, MAP was significantly improved in rats that received the combination dose as compared to the

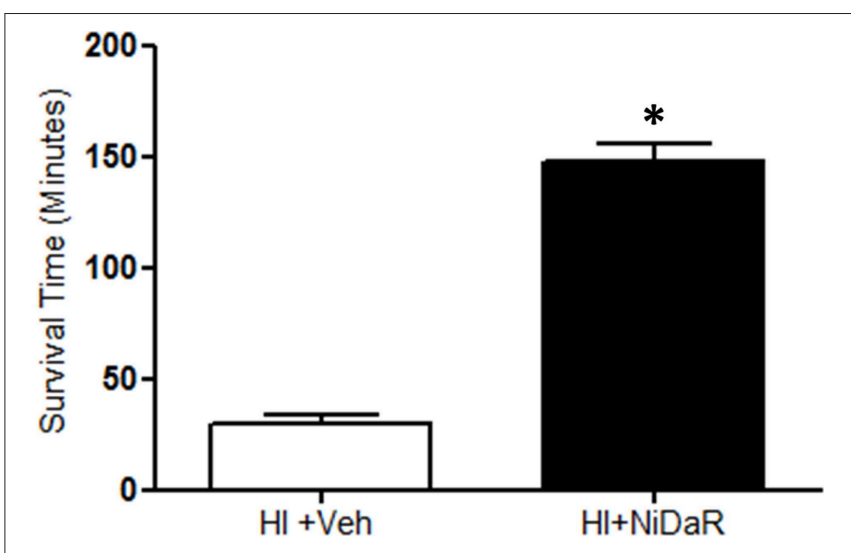

FIGURE 2 | NiDaR prolongs life after hemorrhagic shock. Mean survival time (minutes) following $\mathrm{HI}$ and treatment in each of the experimental groups (mean \pm SEM); $n=5-6$ in each group, *indicates $p<0.05$ compared to $\mathrm{HI}+$ Veh. $\mathrm{NiDaR}$ ( $2 \mathrm{mg} / \mathrm{Kg}$ of each constituent) was administered immediately following shock period.

vehicle treated animals. A significant improvement was observed at the end of fluid resuscitation, as well as at 1 and $2 \mathrm{~h}$ after the end of resuscitation. As expected, blood lactate levels were significantly increased after HI in vehicle treated groups and decreased in $\mathrm{NiDaR}$ treated animals compared to veh group (Figure 3B).

\section{NiDaR Reduces Inflammation Following HI}

Furthermore, NIDaR treatment showed a significant effect on plasma IL-6 levels, the levels were markedly elevated in the untreated group whereas the combination dose was effective in reducing IL-6 in the plasma (Figure 4A). The expression of a number cytokine genes were tested in the heart tissue isolated from the rats subjected to Sham or HI. The expression of cytokines genes IL-6, IL-10, IL-18, and IL-1 $\beta$ were significantly elevated in the heart of rats subjected to HI, and were significantly decreased in NiDaR treated group compared to the Veh treated group (Figure 4B). Consistent with the reduction of inflammatory markers following $\mathrm{HI}$, the phosphorylated NF-kb P65 subunit as well as NLRP3 showed a significant reduction after $\mathrm{HI}$ in NiDaR treated animals (Figures $\mathbf{5 A - C}$ ).

\section{Effect of NiDaR Treatment on Autophagy}

In order to understand the mechanism of $\mathrm{NiDaR}$ induced salutary effect, we investigated the effect of $\mathrm{NiDaR}$ on autophagy. HI was followed by activation of mTOR and NiDaR attenuated mTOR activation, mTOR inhibits autophagy (Figure 6). Furthermore, HI resulted in a significant reduction in the phosphorylated fraction of ULK, a constituent of Atg1 complex and NiDaR treated rats showed significantly increased p-ULK levels in the heart (Figure 7). Consistent with the increase of $\mathrm{p}$ ULK, a similar increase was also observed for $\mathrm{p}$-beclin suggesting that $\mathrm{NiDaR}$ can increase autophagy process after hemorrhagic shock. Interestingly, LC3 II (A/B) expression did not show significant change in the vehicle treated heart, but P62 expression was increased several fold after HI indicating accumulation 

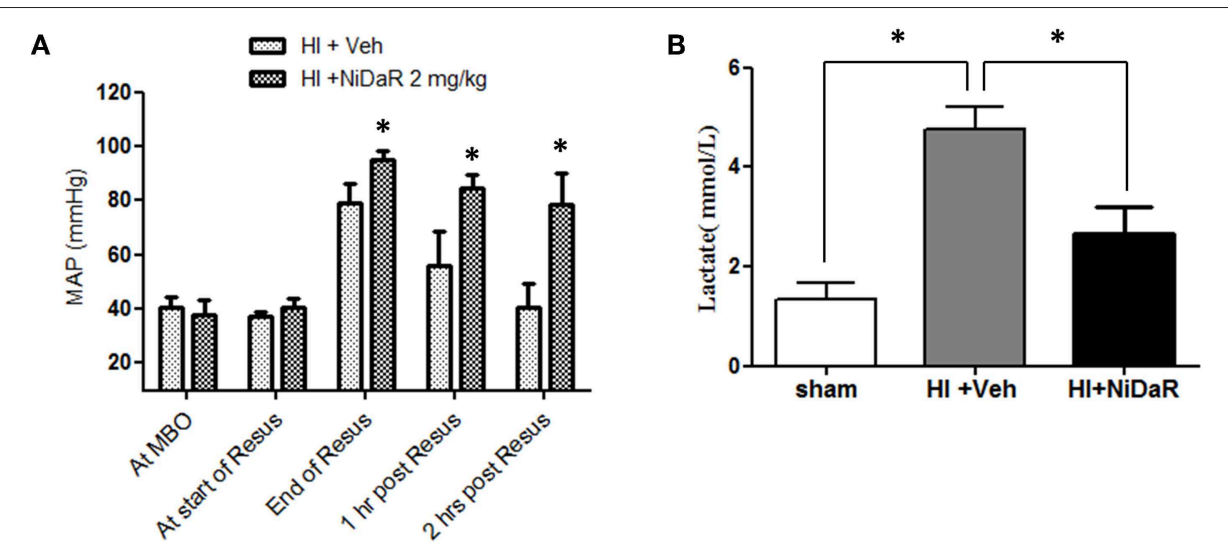

FIGURE 3 | Effect of NiDaR on MAP and plasma lactate following hemorrhagic shock. (A) MAP following HI and treatment with NiDaR at maximum bleed out (MBO), start of resuscitation, end of resuscitation, 1 and $2 \mathrm{~h}$ post resuscitation. Bars represent mean $\pm \mathrm{SEM}$. ${ }^{\star} \mathrm{p}<0.05 \mathrm{vs}$. HI $+\mathrm{Veh}$. (B) Plasma lactate levels in sham, $\mathrm{HI}+$ Veh, $\mathrm{HI}+\mathrm{NiDaR}$ treatment groups; $n=5-6$ in each group; bars represent mean $\pm \mathrm{SEM}$; ${ }^{*}$ indicates $p<0.05$.

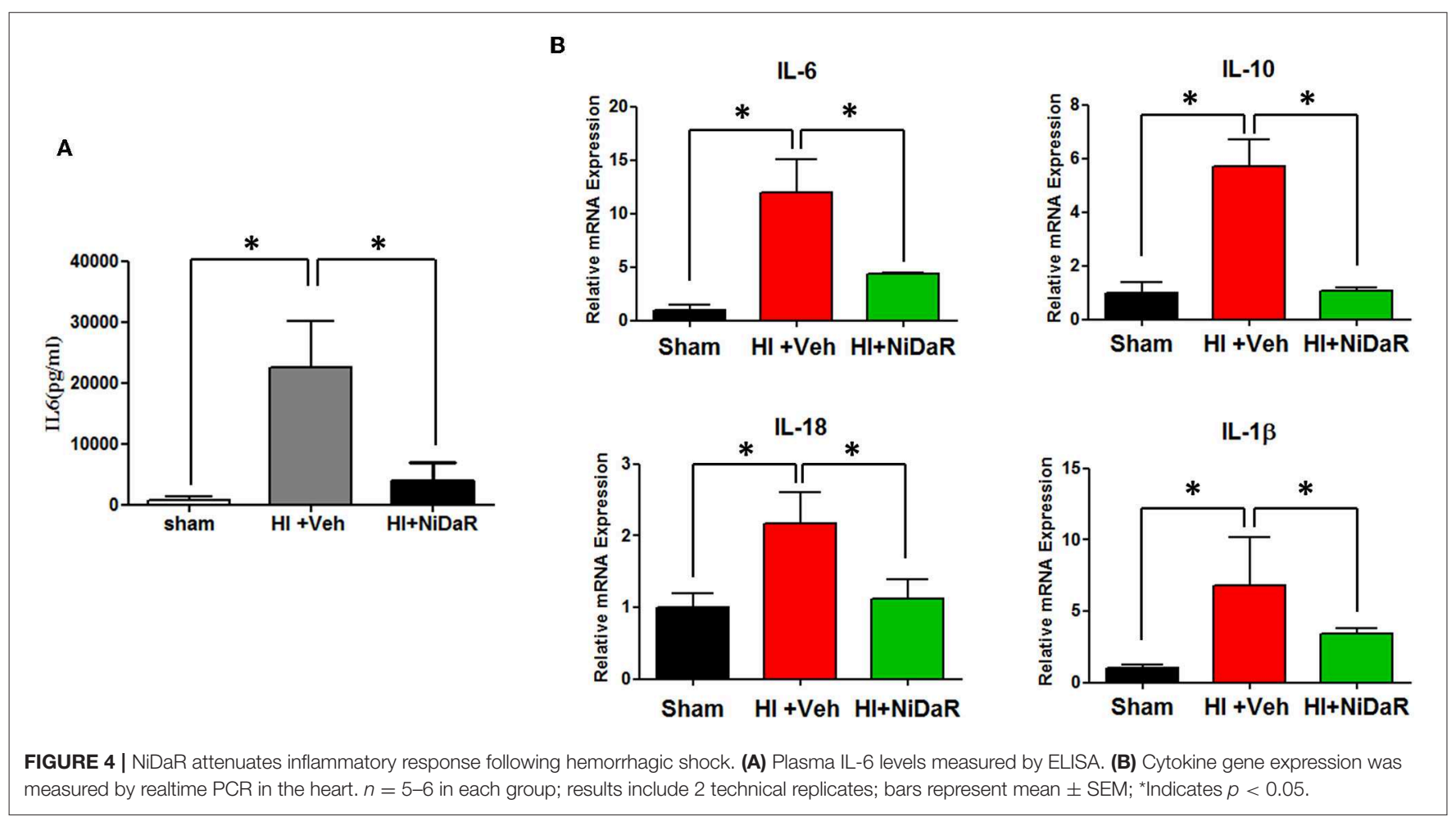

of autophagy substrate with hemorrhagic shock (Figure 8). Whereas, LC3 II expression continued to increase after treatment in NiDaR treated rats, $\mathrm{P} 62$ expression demonstrated a significant decrease with the treatment demonstrating activation of autophagy by the combination dose.

\section{DISCUSSION}

We recently reported in separate papers that niacin, dichloroacetate, and resveratrol have salutary effect in hemorrhagic shock when administered individually at
10-25 mg/Kg dose each $(5,6,15)$. All three compounds directly or indirectly potentiate mitochondrial function and autophagy. In this study we combined all the three compounds to activate multiple pathways and reduce the dose of individual constituents. Furthermore, clinically, such combination doses may be more protective in genetically heterogeneous population. We now demonstrate that when resveratrol, niacin, and dichloroacetate $(\mathrm{NiDaR})$ were combined as a single treatment dose, effective dose of each of these compounds is decreased at least 5-fold. At this lower dose, $\mathrm{NiDaR}$ showed significant improvement in survival, decreased systemic and organ-specific inflammation and enhanced autophagic process. In previous 
A
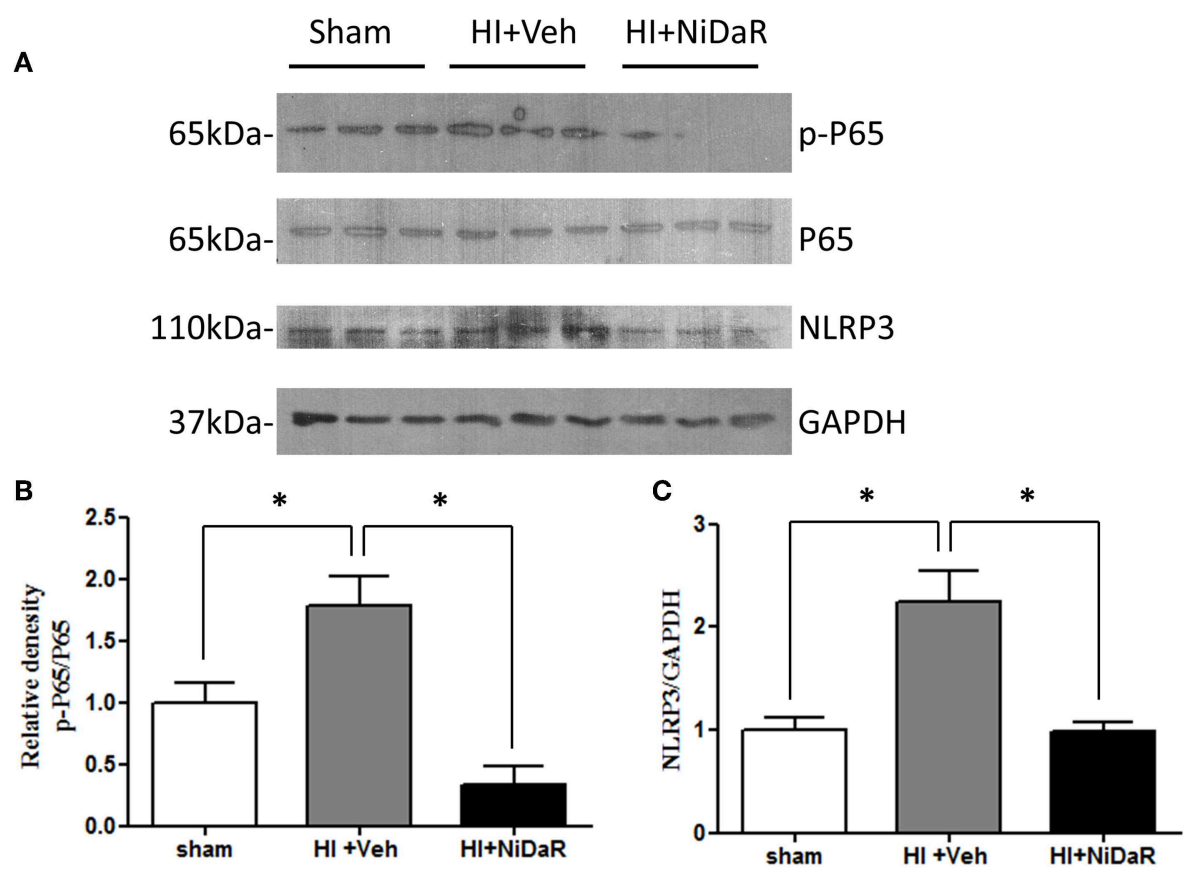

FIGURE 5 | NiDaR reduces NF-kb and NLRP3 levels following hemorrhagic shock. (A) Representative immunoblotting of NF-kB P65, p-P65, NLRP3 in heart tissues of sham, $\mathrm{HI}$ and NiDaR treated rats. GAPDH was used as the loading control. (B) NF-kB p-P65, P65 intensities were quantified using Image $\mathrm{J}$ software(NIH). (C) NLRP3 and GAPDH intensities were quantified using Image $\mathrm{J}$ software $(\mathrm{NIH}) . n=6$ animals each group, 2-3 technical replicates. ${ }^{*}$ ndicates $p<0.05$.

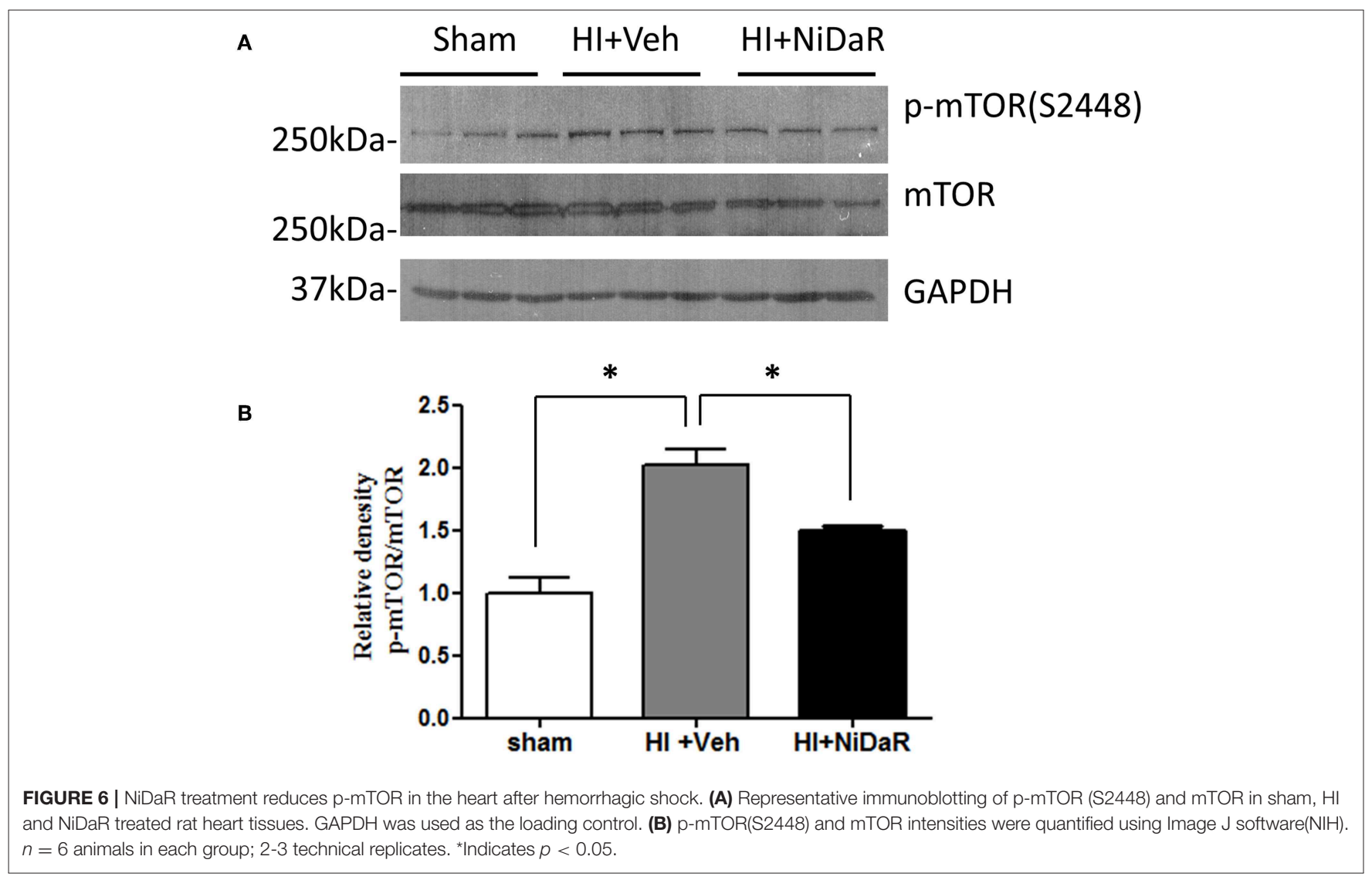




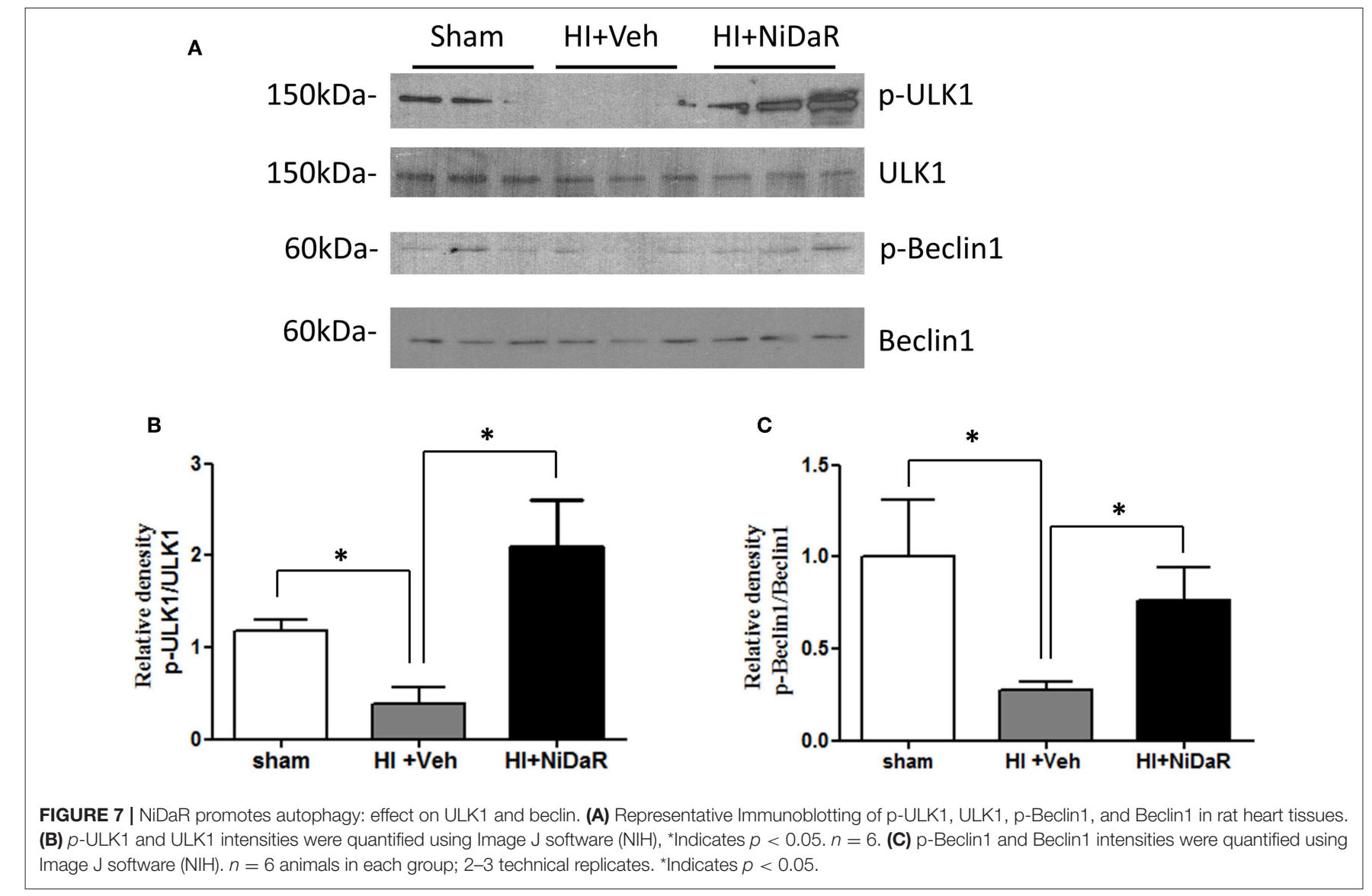

studies $1 \mathrm{mg} / \mathrm{Kg}$ dose of individual compounds did not show significant survival benefit. The effectiveness of $\mathrm{NiDaR}$ was also associated with improved MAP and plasma lactate levels. The use of lower doses of individual compounds may result in reduced side-effects if any, and better effectiveness in a genetically diverse population, in a clinical setting.

A sudden onset of inflammation is a hallmark of hemorrhagic shock (23-26). A significant increase in the level of pro and anti-inflammatory cytokines are observed systemically as well as in several critical organs (27). Administration of $\mathrm{NiDaR}$ as a single dose intravenously along with the resuscitation fluid attenuated the inflammatory response. The IL-6 levels in the plasma as assessed by ELISA was significantly decreased in the animals that received NiDaR. Similarly, the gene expression of IL-6, IL-1 $\beta$, IL-18, and IL-10 in the heart was also attenuated with the combination dose treatment demonstrating effectiveness of the therapy.

A number of immune and metabolic pathways have been previously investigated in hemorrhagic shock by our laboratory and others, and interventions have been proposed based on their effectiveness to control inflammation, improve cellular energetics or reduce cell death $(12,25,28,29)$. Our experiments in this study demonstrate that the cell maintenance process called autophagy is significantly activated in the heart when the animals subjected to $\mathrm{HI}$ were treated with NiDaR. Following $\mathrm{HI}$, there was a significant decline in the phosphorylated form of ULK1 and beclin1 indicating suppression of autophagic process following HI. The nutrient sensor AMPK phosphorylates and activate ULK1 to promote autophagy (30). In a previous study we observed a decrease in the phosphorylation of AMPK following hemorrhagic shock and resuscitation (6). It is wellrecognized that AMPK phosphorylation precedes an energy deprived state, however it is likely that acute injury with a sustained and prolonged reduction in ATP generation and mitochondrial function, as seen in hemorrhagic shock model, leads to reduced phospho-AMPK. Nevertheless, treatment of rats subjected to $\mathrm{HI}$ with a mitochondrial targeted agent, dichloroacetate, increased the p-AMPK/AMPK ratio in the heart, after HI, though the mechanism is unclear (6). ULK1 is an essential component of the Atg1 complex and phosphorylation is critical to its activity $(30,31)$. The significant decline in p-ULK1 observed following HI may be concomittant with the reduced p-AMPK levels thereby inhibiting an effective Atg1 complex formation. The increase in p-ULK1 after treatment with NiDaR indicates enhanced autophagy leading to improved survival. The activation of ULK1 by phosphorylation is a pre-requisite to initiate autophagy (32). ULK1 directly phosphorylates beclin 1 which is an important constituent of VSP34 complex (33), other entities in the complex are also regulated by ULK1 by direct phosphorylation (31). Our studies show a decrease in pbeclin1 in HI, along with the reduced p-ULK1 levels indicating a decrease in autophagic process with HI. Furthermore, treatment with NiDaR promoted autophagy as indicated by decreased $\mathrm{p}$-mTOR, and increase in phosphorylated forms of both 


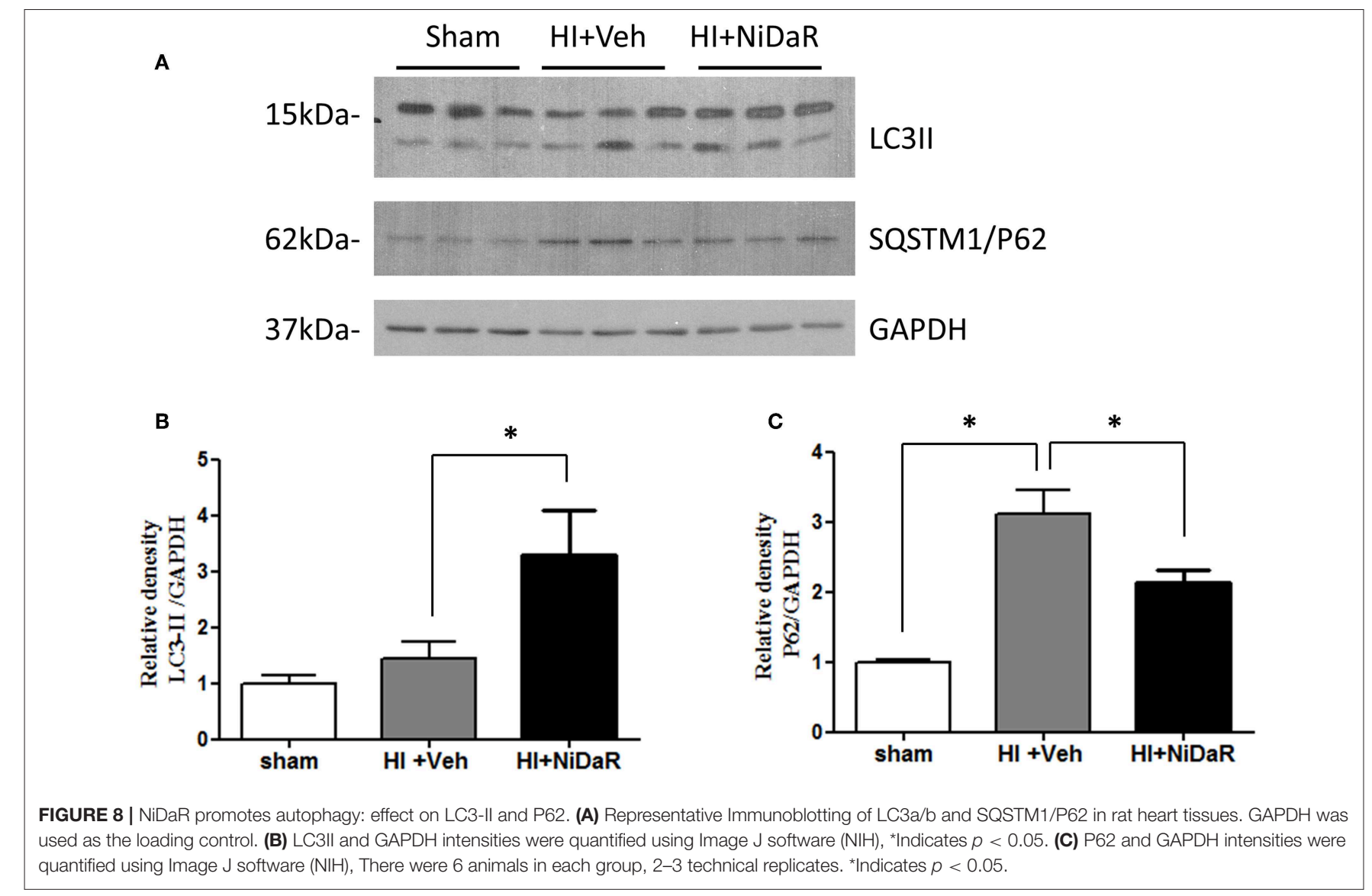

ULK1 and Beclin1 in the heart of groups of rats treated with $\mathrm{NiDaR}$.

A decrease in the level of any of the components involved in Atg1 complex results in reduced autophagic clearance and accumulation of autophagic substrates such as P62 (34). The protein level of P62 in the heart was found to increase about 3fold following $\mathrm{HI}$ and this may indicate a disrupted autophagy. However, treatment with the combination dose demonstrated a significant decrease in P62 levels demonstrating acceleration of autophagy. The LC3-II levels after HI remained the same as basal level indicating no significant increase in autophagic activity at $2 \mathrm{~h}$ after resuscitation, whereas the levels were significantly increased following $\mathrm{NiDaR}$ treatment, an indication of increased formation of autophagosomes. Nevertheless, the significant increase of LC3-II in the animals that were treated with the combination dose may indicate either an increased autophagy or inhibition of autophagosome degradation as LC3-II amount at a given time point does not necessarily estimate the autophagic activity (34). The latter cause may be ruled out considering the significant decrease in autophagic susbtrate (P62) with NiDaR treatment. However, the NiDaR group showed larger changes than maintaining levels observed in shams, it is not clear whether this is a dose effect or whether there could be consequences of such alterations in the treatment group.
One of the limitations of the study was that we administered only a single dose of $\mathrm{NiDaR}$ after hemorrhagic shock. The effect of administering $\mathrm{NiDaR}$ at multiple intervals or continuous infusion as was done in the clinical trial (CRASH-2) of tranexamic acid (35) needs to determined. In order to transition to clinically relevant studies, it is also necessary to test the effectiveness of $\mathrm{NiDaR}$ in long term survival with fluid resuscitation. Small volume treatments such as $\mathrm{NiDaR}$ can be given as first line treatment before resuscitation fluid could be available. The survival study in which we did not perform fluid resuscitation addresses this aspect.

Our experiments demonstrate lack of enhanced autophagic activity or inhibition of autophagy with $\mathrm{HI}$ and promotion of autophagic activity when treated with NiDaR. The positive regulation of autophagy by $\mathrm{NiDaR}$ in $\mathrm{HI}$ is not surprising as niacin, dichloroactetate, and resveratrol have been shown to independently activate autophagy in other models $(17,19,36)$, however this is the first systematic demonstration of autophagic regulation following HI. In summary, the study shows that $\mathrm{NiDaR}$ attenuates inflammation and promote autophagy following hemorrhagic shock and this combinatorial formulation is promising in the treatment of hemorrhagic shock. 


\section{DATA AVAILABILITY STATEMENT}

The datasets generated for this study are available on request to the corresponding author.

\section{ETHICS STATEMENT}

All animal experiments were approved by the Institutional Animal Care and Use Committee (IACUC) at Augusta University and were performed in adherence to the NIH Guidelines on the Use of Laboratory Animals. Male Sprague Dawley rats of ages 10-12 weeks were obtained from Charles River Laboratory (Wilmington, MA, USA).

\section{REFERENCES}

1. DiMaggio C, Ayoung-Chee P, Shinseki M, Wilson C, Marshall G, Lee DC, et al. Traumatic injury in the United States: in-patient epidemiology 2000-2011. Injury. (2016) 47:1393-403. doi: 10.1016/j.injury.2016.04.002

2. Hooper N, Armstrong TJ. Hemorrhagic Shock. In: StatPearls [Internet]. Treasure Island, FL: StatPearls Publishing (2019).

3. Kauvar DS, Lefering R, Wade CE. Impact of hemorrhage on trauma outcome: an overview of epidemiology, clinical presentations, and therapeutic considerations. J Trauma. (2006) 60:S3-11. doi: 10.1097/01.ta.0000199961.02677.19

4. Bougle, Harrois A, Duranteau J. Resuscitative strategies in traumatic hemorrhagic shock. Ann Intensive Care. (2013) 3:1. doi: 10.1186/2110-5820-3-1

5. Ayub, Poulose N, Raju R. Resveratrol improves survival and prolongs life following hemorrhagic shock. Mol Med. (2015) 21:305-12. doi: 10.2119/molmed.2015.00013

6. Subramani K, Lu S, Warren M, Chu X, Toque HA, Caldwell RW, et al. Mitochondrial targeting by dichloroacetate improves outcome following hemorrhagic shock. Sci Rep. (2017) 7:2671. doi: 10.1038/s41598-017-02495-5

7. Ham PB III, Raju R. Mitochondrial function in hypoxic ischemic injury and influence of aging. Prog Neurobiol. (2017) 157:92-116. doi: 10.1016/j.pneurobio.2016.06.006

8. Poulose N, Raju R. Aging and injury: alterations in cellular energetics and organ function. Aging Dis. (2014) 5:101-8. doi: 10.1016/j.bbadis.2015.08.017

9. Warren M, Subramani K, Schwartz R, Raju R. Mitochondrial dysfunction in rat splenocytes following hemorrhagic shock. Biochim Biophys Acta. (2017) 1863:2526-33. doi: 10.1016/j.bbadis.2017.08.024

10. Muller T, Kirsch M, Petrat F. Attenuation of multiple organ damage by continuous low-dose solvent-free infusions of resveratrol after severe hemorrhagic shock in rats. Nutrients. (2017) 9:889. doi: 10.3390/nu9080889

11. Powell RD, Swet JH, Kennedy KL, Huynh TT, McKillop IH, Evans SL. Resveratrol attenuates hypoxic injury in a primary hepatocyte model of hemorrhagic shock and resuscitation. J Trauma Acute Care Surg. (2014) 76:409-17. doi: 10.1097/TA.0000000000000096

12. Sims CA, Baur JA. The grapes and wrath: using resveratrol to treat the pathophysiology of hemorrhagic shock. Ann N Y Acad Sci. (2017) 1403:7081. doi: $10.1111 /$ nyas. 13444

13. Wang H, Guan Y, Karamercan MA, Ye L, Bhatti T, Becker LB, et al. Resveratrol rescues kidney mitochondrial function following hemorrhagic shock. Shock. (2015) 44:173-80. doi: 10.1097/SHK.0000000000000390

14. Liu FC, Tsai YF, Tsai HI, Yu HP. Anti-inflammatory and organ-protective effects of resveratrol in trauma-hemorrhagic injury. Mediat Inflamm. (2015) 2015:643763. doi: 10.1155/2015/643763

15. Subramani K, Chu X, Warren M, Lee M, Lu S, Singh N, et al. Deficiency of metabolite sensing receptor HCA2 impairs the salutary effect of niacin in hemorrhagic shock. Biochim Biophys Acta Mol Basis Dis. (2019) 1865:68895. doi: 10.1016/j.bbadis.2019.01.009

16. Canto, Menzies KJ, Auwerx J. NAD $(+)$ metabolism and the control of energy homeostasis: a balancing act between mitochondria and the nucleus. Cell Metab. (2015) 22:31-53. doi: 10.1016/j.cmet.2015.05.023

\section{AUTHOR CONTRIBUTIONS}

XC designed, performed, and interpreted most of the experiments. RR took part in conceptualization, planning, interpretation of the data, and edited and finalized the manuscript. RS and $\mathrm{MD}$ participated in the interpretation of the data, editing, and finalizing the manuscript.

\section{FUNDING}

This study was supported by the NIH grant R01 GM122059 (RR).

17. Kim SW, Lee JH, Moon JH, Nazim UM, Lee YJ, Seol JW, et al. Niacin alleviates TRAIL-mediated colon cancer cell death via autophagy flux activation. Oncotarget. (2016) 7:4356-68. doi: 10.18632/oncotarget.5374

18. Li B, Li X, Ni Z, Zhang Y, Zeng Y, Yan X, et al. Dichloroacetate and metformin synergistically suppress the growth of ovarian cancer cells. Oncotarget. (2016) 7:59458-70. doi: 10.18632/oncotarget.10694

19. Lin G, Hill DK, Andrejeva G, Boult JK, Troy H, Fong AC, et al. Dichloroacetate induces autophagy in colorectal cancer cells and tumours. $\mathrm{Br}$ J Cancer. (2014) 111:375-85. doi: 10.1038/bjc.2014.281

20. Morselli E, Maiuri MC, Markaki M, Megalou E, Pasparaki A, Palikaras $\mathrm{K}$, et al. Caloric restriction and resveratrol promote longevity through the Sirtuin-1-dependent induction of autophagy. Cell Death Dis. (2010) 1:e10. doi: $10.1038 /$ cddis. 2009.8

21. Qu X, Chen X, Shi Q, Wang X, Wang D, Yang L. Resveratrol alleviates ischemia/reperfusion injury of diabetic myocardium via inducing autophagy. Exp Ther Med. (2019) 18:2719-25. doi: 10.3892/etm.2019.7846

22. Zhang DX, Zhang JP, Hu JY, Huang YS. The potential regulatory roles of $\mathrm{NAD}(+)$ and its metabolism in autophagy. Metabolism. (2016) 65:45462. doi: 10.1016/j.metabol.2015.11.010

23. Aziz M, Brenner M, Wang P. Extracellular CIRP (eCIRP) and inflammation. J Leukoc Biol. (2019) 106:133-46. doi: 10.1002/JLB.3MIR1118-443R

24. Zingarelli B, Sheehan M, Wong HR. Nuclear factor-kappaB as a therapeutic target in critical care medicine. Critic Care Med. (2003) 31:S10511. doi: 10.1097/00003246-200301001-00015

25. Albertsmeier M, Pratschke S, Chaudry I, Angele MK. Gender-specific effects on immune response and cardiac function after trauma hemorrhage and sepsis. Viszeralmedizin. (2014) 30:91-6. doi: 10.1159/000360149

26. Raju R. Immune and metabolic alterations following trauma and sepsis - an overview. Biochim Biophys Acta Mol Basis Dis. (2017) 1863:25235. doi: 10.1016/j.bbadis.2017.08.008

27. Lu S, Aguilar A, Subramani K, Poulose N, Ayub A, Raju R. Alteration of cytokine profile following hemorrhagic shock. Cytokine. (2016) 81:358. doi: 10.1016/j.cyto.2016.01.022

28. Clendenen N, Nunns GR, Moore EE, Reisz JA, Gonzalez E, Peltz E, et al. Hemorrhagic shock and tissue injury drive distinct plasma metabolome derangements in swine. J Trauma Acute Care Surg. (2017) 83:63542. doi: 10.1097/TA.0000000000001504

29. Xie Y, Xu M, Deng M, Li Z, Wang P, Ren S, et al. Activation of pregnane $\mathrm{X}$ receptor sensitizes mice to hemorrhagic shock-induced liver injury. Hepatology. (2019) 70:995-1010. doi: 10.1002/hep.3069

30. Roach PJ. AMPK -> ULK1 -> autophagy. Mol Cell Biol. (2011) 31:30824. doi: 10.1128/MCB.05565-11

31. Zachari M, Ganley IG. The mammalian ULK1 complex and autophagy initiation. Essays Biochem. (2017) 61:585-96. doi: 10.1042/EBC20170021

32. Wang Z, Wilson WA, Fujino MA, Roach PJ. Antagonistic controls of autophagy and glycogen accumulation by Snflp, the yeast homolog of AMPactivated protein kinase, and the cyclin-dependent kinase Pho85p. Mol Cell Biol. (2001) 21:5742-52. doi: 10.1128/MCB.21.17.5742-5752.2001

33. Russell RC, Tian Y, Yuan H, Park HW, Chang YY, Kim J, et al. ULK1 induces autophagy by phosphorylating Beclin-1 and activating VPS34 lipid kinase. Nat Cell Biol. (2013) 15:741-50. doi: 10.1038/ncb2757 
34. Yoshii SR, Mizushima N. Monitoring and measuring autophagy. Int J Mol Sci. (2017) 18:1865. doi: 10.3390/ijms180 91865

35. CRASH-2 collaborators, Roberts I, Shakur H, Afolabi A, Brohi K, Coats T, et al. The importance of early treatment with tranexamic acid in bleeding trauma patients: an exploratory analysis of the CRASH-2 randomised controlled trial. Lancet. (2011) 377:1096-101. doi: 10.1016/S0140-6736(11) 60278-X

36. Ren J, Zhang Y. Targeting autophagy in aging and agingrelated cardiovascular diseases. Trends Pharmacol Sci. (2018) 39:1064-76. doi: 10.1016/j.tips.2018.10.005
Conflict of Interest: The authors declare that the research was conducted in the absence of any commercial or financial relationships that could be construed as a potential conflict of interest.

Copyright (C) 2019 Chu, Schwartz, Diamond and Raju. This is an open-access article distributed under the terms of the Creative Commons Attribution License (CC BY). The use, distribution or reproduction in other forums is permitted, provided the original author(s) and the copyright owner(s) are credited and that the original publication in this journal is cited, in accordance with accepted academic practice. No use, distribution or reproduction is permitted which does not comply with these terms. 